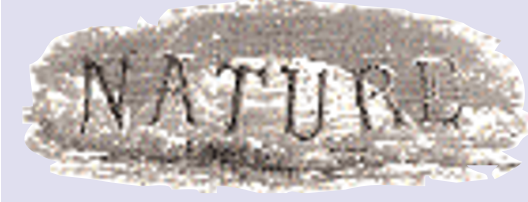

100 YEARS AGO

The widespread invasion and persistent devastations of locusts in so many parts of Africa give interest to all trials and experiments, as well as the ordinary remedies, employed for the alleviation of this ruinous plague of the farmer. The following notes from Mr. W. C. Robbins, Stock Inspector of the Lower Tugela and Mapumulo Districts, are published in the Cape official Agricultural Journal:- "For the past three days I have been over the ground where my men have been infecting locusts with Government fungus, and the result was that I found dead locusts everywhere. I send you a sample; you will notice they are full of worms, and we know from experience that when locusts are found in this state whole swarms die off. Some you will see, are half eaten; these were eaten by their fellows. I have seen many clusters of locusts eating dead ones." The feeding upon bodies of dead locusts suggests that diseased locusts may be utilised as a substitute for locust fungus. From Nature 7 June 1900.

\section{YEARS AGO}

The gestural or 'ta-ta' theory of the origin of language has a long pedigree, beginning with Plato and achieving its most notable modern exponent in Sir Richard Paget. That Prof. Jóhannesson had been attracted to this theory was evident from his earlier work, " $\mathrm{Um}$ Frumtungu Indógermana og Frumheimkynni"; and the dedication of this new volume to Sir Richard sets the author decisively among those who believe themselves capable of demonstrating that language originated in the imitation by the vocal organs of physical shapes and movement. This theory represents one branch of the more general doctrine which sees a natural connexion between things and their names: and it is not to be denied that certain sound-complexes seem, in fact, to be particularly appropriate to the representation of certain shapes - a field of study in which Koehler and the Gestalt psychologists have collected some interesting data. But even supposing that these soundcomplexes could be shown to involve vocal movements related to the shapes concerned, this would teach us nothing about the origin of language, which must be far remote from the attested or 'reconstructed' forms on which the theory is based - though Prof. Jóhannesson gives one the impression that he believes his hypothetical Indo-European forms at least to approximate to a primitive tongue. From Nature 10 June 1950. two polaritons with the same quantum wavenumber are simultaneously created by a coherent laser pulse (the 'pump' pulse). These two polaritons are scattered to different energy states - the ground state and a higher-energy state - in a way that conserves energy and momentum between the initial and final states. When the groundstate polariton is stimulated by another coherent laser pulse, an enormous gain of up to 100 is observed.

This might seem like a matter-wave analogue of a laser amplifier. However, it does not correspond to a laser amplifier in an exact sense, but rather to a 'parametric' amplifier. In the radiowave, microwave and opticalwave spectrum, there are always two types of amplifier: a negative-conductance amplifier and a nonlinear-susceptance amplifier. A negative-conductance amplifier is an incoherent system, in which only the energy of the pump pulse determines the system dynamics. A laser amplifier is a classic example of a negative-conductance amplifier. A nonlinear-susceptance amplifier is a coherent system in which both the amplitude and the phase of the pump pulse determine the system dynamics. A parametric amplifier is a classic example of a nonlinear-susceptance amplifier. In the experiment of Savvidis et $a l^{1}{ }^{1}$, the ground-state polariton is amplified by a phase-coherent parametric process.

In a different study, Huang et al. ${ }^{2}$ create a direct matter-wave analogue of a laser amplifier using essentially the same structure as Savvidis et al. In this experiment, two reservoir polaritons with opposite quantum wavenumbers are scattered to the ground and excited states with zero wavenumber. Amplification is observed even after the reservoir polaritons lose the phase information of the pump laser and reach thermal equilibrium. This means that matter-wave amplification in this system could be achieved using electrically injected incoherent excitons.

Matter-wave amplifiers using atoms $\mathrm{s}^{3,4}$ rather than excitons operate on the same principle, but their working conditions are quite different. The effective temperature of an exciton reservoir is about $10 \mathrm{~K}$, whereas that of an atomic reservoir (Bose-Einstein condensate) is about $10 \mathrm{nK}$. The gain and loss rates of an exciton matter-wave amplifier are approximately $10^{12} \mathrm{~s}^{-1}$, whereas those of an atomic matter-wave amplifier are approximately $10^{5} \mathrm{~s}^{-1}$. The ratio of the average spacing between two excitons compared to their radius is about 30 , whereas that of the average spacing between two atoms to the atomic radius is about $10^{3}$. Exciton matterwave amplifiers operate at a much higher temperature, gain and particle density than their atomic counterparts.

The exciton laser and amplifier can operate with a much smaller pump power than a normal photon laser. A photon laser and amplifier requires more populations in an

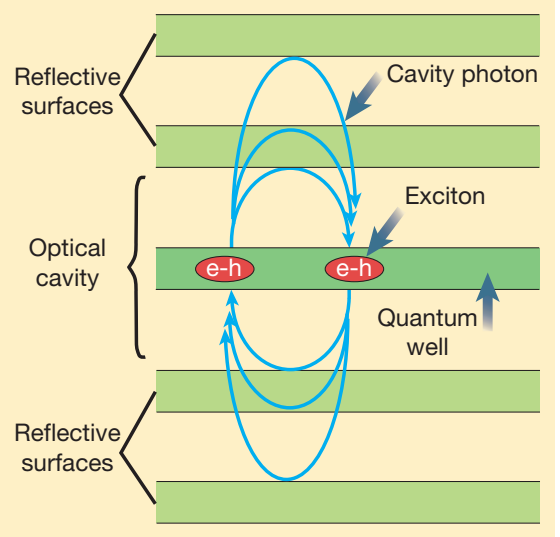

Figure 1 A semiconductor matter-wave amplifier. Here, a semiconductor quantum well is sandwiched between two reflective microcavities, which are separated by one-half or one wavelength. An exciton, which is a bound state of an electron-hole (e-h) pair and the solid-state analogue of a hydrogen atom, is confined in the quantum well by the potential barrier between the quantum well and the surrounding materials. A light field is trapped by multiple reflection from the reflective interfaces. When a quantum-well exciton and a cavity photon couple strongly with each other, they form a new quasiparticle called a polariton. Savvidis et al. ${ }^{1}$ show that the polaritons can be excited resonantly by a laser producing amplification of the signal.

excited state than in the ground state (socalled population inversion). But the exciton laser and amplifier operate without inver$\operatorname{sion}^{5}$. A wide bandgap semiconductor, such as gallium nitride or zinc oxide, is more suitable for constructing such an exciton laser and amplifier because the excitons in such materials are stable at higher temperatures and densities. Such semiconductor lasers are attractive because they are an efficient source of blue light.

If the gain of a matter-wave amplifier exceeds the loss rate, the system is expected to form a matter-wave laser (boser) ${ }^{5}$. But the spontaneous build-up of a coherent matter wave without continuous pumping by an input matter wave has yet to be seen. Photoluminescence studies of microcavities in semiconductor quantum wells ${ }^{6,7}$ suggest that this goal is within the reach of current technology.

Yoshihisa Yamamoto is at the Quantum Entanglement Project, Edward L. Ginzton Laboratory, Stanford University, Stanford, California 94305-4085, USA, and NTT Basic Research Laboratories, Atsugishi, Kanagawa, Japan. e-mail:yamamoto@loki.stanford.edu

1. Savvidis, P. et al. Phys. Rev. Lett. 84, 1547-1550 (2000).

2. Huang, R. et al. Phys. Rev. B 61, R7854-R7857 (2000).

3. Inouye, S. et al. Nature 402, 641-644 (1999).

4. Helmerson, K. Nature 402, 587-588 (1999).

5. Imamoglu, A. et al. Phys. Rev. A 53, 4250-4253 (1996).

6. Dang, L. S. et al. Phys. Rev. Lett. 81, 3920-3923 (1998).

Senellart, P. \& Bloch, J. Phys. Rev. Lett. 82, 1233-1236 (1999). 